\title{
TILES WITH NO SPECTRA IN DIMENSION 4
}

\author{
BÁLINT FARKAS and SZILÁRD GY. RÉVÉSZ*
}

\begin{abstract}
We show by a counterexample that the "tiling $\Rightarrow$ spectral" part of Fuglede's Spectral Set Conjecture
\end{abstract} fails already in $Z^{4}$ and $R^{4}$.

\section{Introduction}

The Spectral Set Conjecture of Fuglede [1] relates the class of tiling sets of $\mathrm{R}^{d}$ to some Fourier analytic property, called spectrality. To be able to state the conjecture precisely we recall the appropriate setting. Let $G$ be a locally compact Abelian group (we will only consider $\mathrm{Z}^{d}, \mathrm{R}^{d}$ and finite commutative groups), the dual group is denoted by $\widehat{G}$. Once for all we fix a Haar-measure on $G$, and $\widehat{f}$ will stand for the Fourier transform of a function $f: G \rightarrow \mathrm{C}$. $Z(f)$ denotes the zero set of the function $f$. Further we use the notation $\chi_{T}$ for the characteristic function of the set $T \subseteq G$.

Definition. An open set $T \subseteq G$ is called spectral with spectrum $L \subseteq \widehat{G}$ if $L$ is a complete orthogonal system in $L_{2}(T)$.

Definition. An open subset $T$ of $G$ is said to be a tiling set (or simply tile), if the whole group $G$ can be covered by translated disjoint copies of $T$ up to a set of zero measure. That is there exists a set $T^{\prime} \subseteq G$, called a tiling complement of $T$ such that $T^{\prime}+T$ is the whole of $G$ except a set of zero measure and for all $t \neq s, t, s \in T^{\prime}$ we have $(t+T) \cap(s+T)=\emptyset$.

REMARK 1. It is easy to see - and will be used throughout - that the latter packing condition is equivalent to $(T-T) \cap\left(T^{\prime}-T^{\prime}\right)=\{0\}$. In fact, for a finite group $G$ tiling is equivalent to $|G|=|T| \cdot\left|T^{\prime}\right|$ and $(T-T) \cap\left(T^{\prime}-T^{\prime}\right)=\{0\}$.

Now, the Spectral Set Conjecture reads as follows.

A domain $\Omega \subseteq \mathrm{R}^{d}$ is spectral if and only if it can tile $\mathrm{R}^{d}$ by translations.

* Supported by the Hungarian National Foundation for Scientific Research, Grant \# T-049693 and T-049301, and by the Hungarian-French Scientific and Technological Governmental Cooperation, Project \# F-10/04.

Received January 10, 2005. 
Although there were many results supporting the conjecture (already Fuglede himself proved it in case the tiling complement or the spectrum is assumed to be a lattice), Tao [15] has recently come up with a counterexample, disproving the "spectral $\Rightarrow$ tiling" part in dimension 5 and higher. Matolcsi [11] has reduced this dimension to 4, and later Kolountzakis and Matolcsi [6] disproved this part in dimension 3. They also clarified a method that could be used to give counterexamples in lower dimensions. Concerning the other, "tiling $\Rightarrow$ spectral" direction of the conjecture Kolountzakis and Matolcsi [7] have given a counterexample in dimension larger or equal to 5. Our aim is to prove

Theorem 1. There exists a tiling set in $\mathrm{R}^{4}$ which is not spectral.

The constructions of Tao [15] and Kolountzakis, Matolcsi [7] are based on examples in finite commutative groups. Let us describe the now automatic transition mechanism of transferring a counterexample from a finite Abelian group to $Z^{d}$ and $\mathrm{R}^{d}$ by quoting the following two results of Kolountzakis and Matolcsi from [7]. (Hereafter $Z_{n}$ denotes the cyclic group of $n$ elements, for convenience regarded as $\mathbf{Z} / n \mathbf{Z}$.)

Theorem 2 (Kolountzakis-Matolcsi). Let $\mathbf{n}=\left(n_{1}, \ldots, n_{d}\right) \in \mathrm{N}^{d}$ and consider a set $A \subseteq G=Z_{n_{1}} \times \cdots \times Z_{n_{d}}$. For the set

(1)

$T=T(\mathbf{n}, k)=\left\{0, n_{1}, 2 n_{1}, \ldots,(k-1) n_{1}\right\} \times \cdots \times\left\{0, n_{d}, 2 n_{d}, \ldots,(k-1) n_{d}\right\}$

define $B(k)=A+T$. Then, for large enough values of $k$, the set $B(k) \subset Z^{d}$ is spectral in $\mathrm{Z}^{d}$ if and only if $A$ is spectral in $G$.

Theorem 3 (Kolountzakis-Matolcsi). Suppose $B \subseteq \mathrm{Z}^{d}$ is a finite set and $Q=(0,1)^{d}$. Then $B$ is a spectral set in $Z^{d}$ if and only if $B+Q$ is a spectral set in $\mathrm{R}^{d}$.

Note that obviously in the above constructions for a tile $A \subset G$ we must also have that $B=B(k) \subset \mathrm{Z}^{d}$ tiles $\mathrm{Z}^{d}$ (for any $k \in \mathrm{N}$ ) and for $B \subset \mathrm{Z}^{d}$ tiling $\mathrm{Z}^{d}$ also $B+Q$ tiles $\mathrm{R}^{d}$. Whence it is now straightforward that our task is reduced to exhibit a counterexample in a finite group $G$.

\section{Proof of the result}

We are going to prove Theorem 1 at the end of this section. First we start by constructing a counterexample in a finite group, which indeed suffices, as described in the introduction.

To exhibit a counterexample in $\mathrm{R}^{4}$, we follow the idea of Kolountzakis and Matolcsi [7], which is based on arguments in $Z_{6}^{5}$ and the extension of the finite counterexamples to $Z^{5}$ and $R^{5}$. However, to go down with the dimension to 4 , 
we have to modify the starting point, and to construct an example of "tiling $\nRightarrow$ spectral" first in the group $Z^{4}$, based on considerations in $Z_{6}^{4}$.

When working with $d \times r$ matrices over a finite commutative group $G$, the column and row vectors are regarded as elements of $G^{d}$ and $\widehat{G^{r}}$, respectively. Particularly for cyclic groups $G=\mathrm{Z}_{n}$ the duality pairing between $G^{d}$ and $\widehat{G^{d}}$ in this identification takes the following form

$$
\gamma(g)=e^{\frac{2 \pi i}{n} \gamma \cdot g} \quad \text { for } \quad g \in G^{d}=Z_{n}^{d}, \gamma \in \widehat{G^{d}}=\left(Z_{n}^{d}\right)^{\top} .
$$

We will also "identify" any matrix with the set of its columns or rows; the meaning should be obvious from the context. For example, consider the mod 6 matrices

$$
T:=\left(\begin{array}{cccccc}
0 & 1 & 0 & 0 & 0 & 2 \\
0 & 0 & 1 & 0 & 0 & 2 \\
0 & 0 & 0 & 1 & 0 & 2 \\
0 & 0 & 0 & 0 & 1 & 2
\end{array}\right) \quad \text { and } \quad L:=\left(\begin{array}{llll}
0 & 0 & 0 & 0 \\
0 & 2 & 2 & 4 \\
2 & 0 & 4 & 4 \\
2 & 4 & 0 & 2 \\
4 & 4 & 2 & 0 \\
4 & 2 & 4 & 2
\end{array}\right) .
$$

Then $T \subseteq \mathrm{Z}_{6}^{4}$ is a spectral set with spectrum $L \subseteq \widehat{\mathrm{Z}}_{6}^{4}$. This is so because $L \cdot T=K$ holds $\bmod 6$, with

$$
K:=\left(\begin{array}{cccccc}
0 & 0 & 0 & 0 & 0 & 0 \\
0 & 0 & 2 & 2 & 4 & 4 \\
0 & 2 & 0 & 4 & 4 & 2 \\
0 & 2 & 4 & 0 & 2 & 4 \\
0 & 4 & 4 & 2 & 0 & 2 \\
0 & 4 & 2 & 4 & 2 & 0
\end{array}\right)
$$

and $\frac{1}{6} K$ (considered now as a matrix of real numbers) is a log-Hadamard matrix. (Recall that Matolcsi [11] calls a square matrix $H=\left[h_{j k}\right]_{j, k=1, \ldots, n}$ a $l o g$ Hadamard matrix if the entrywise exponential of $2 \pi i H$, that is, $\left[e^{2 \pi i h_{j k}}\right]_{j, k=1, \ldots, n}$, is a complex Hadamard matrix, i.e., a complex matrix with orthogonal rows and all entries having absolute value 1). The matrix $K$ first appeared in the context of the Spectral Set Conjecture in Tao [15]. Later, Kolountzakis and Matolcsi [7] used it to construct a counterexample to the "tiling $\Rightarrow$ spectral" part of Fuglede's conjecture, and also the above decomposition (originally mod 3) was utilized in [11] to bring down the dimension in the disproof of the other, "spectral $\Rightarrow$ tiling" direction of the conjecture.

In finite groups $G$ there is a very straightforward way of justifying that a subset $T \subseteq G$ is not a tile. Namely, if the number of elements $|T|$ does not divide the order $|G|$ of the group, then $T$ cannot be a tile. Unfortunately we 
have no such immediate evidence for being not spectral. However, a convenient reformulation of being a spectrum is the following.

Proposition 1 (Kolountzakis [5] p. 37, Kolountzakis-Matolcsi [7]). The set $S \subseteq \widehat{G}$ is a spectrum of the set $R \subseteq G$ if and only if $|S|=|R|$ and $S-S \subseteq Z\left(\widehat{\chi}_{R}\right) \cup\{0\}$.

Since we want to find a tile which is not spectral, we will use the above proposition together with a duality argument (see [7]).

Lemma 2. Let $R \subseteq G$ be a subset in $G$ and suppose that there is a subset $L \subseteq \widehat{G}$ with $|R| \cdot|L|=|G|$ such that $L$ is not a tile in $\widehat{G}$ and $Z\left(\widehat{\chi}_{R}\right) \cap(L-L)=$ $\emptyset$. Then $R$ can not be spectral.

Proof. If $S$ was a spectrum of $R$, then $|S|=|R|$ and $S-S \subseteq Z\left(\widehat{\chi}_{R}\right) \cup\{0\}$ in view of Proposition 1, and hence the packing condition $(S-S) \cap(L-L)=\{0\}$ would hold. Since by condition we also have $|\widehat{G}|=|G|=|R| \cdot|L|=|S| \cdot|L|$, this packing condition and Remark 1 ensures that $S+L$ is in fact a tiling of $\widehat{G}$, which is impossible by assumption.

Therefore, ultimately, our goal is to establish the situation presented in the above lemma, i.e., to construct a tiling set $R \subset G$ together with a corresponding $L \subset \widehat{G}$ satisfying the above assumptions.

Remark 2. Suppose that the conditions of Lemma 2 are fulfilled and moreover that $R$ is tiling (this is what we are aiming at). Then for any tiling complement $T$ of $R$ we have $|R| \cdot|T|=|G|$ and also $\chi_{R+T}=\chi_{G}, \widehat{\chi}_{G}=\widehat{\chi}_{R} \cdot \widehat{\chi}_{T}$, thus $Z\left(\widehat{\chi}_{R}\right) \cup Z\left(\widehat{\chi}_{T}\right) \cup\{0\}=\widehat{G}$. Hence the assumption $Z\left(\widehat{\chi}_{R}\right) \cap(L-L)=\emptyset$ leads to $Z\left(\widehat{\chi}_{T}\right) \cup\{0\} \supseteq(L-L)$ and so by $|L|=|G| /|R|=|T|$ we find that $L$ is a spectrum of $T$ according to Proposition 1. That is, $T$ is tiling with complement $R$, and is spectral with spectrum $L$, but also $Z\left(\widehat{\chi}_{R}\right) \cap(L-L)=\emptyset$ is satisfied. This shows that possible examples of $R, T$ and $L$ satisfying the condition in Lemma 2 have to be such that $R$ is tiling with complement $T$ whose spectrum is $L$.

So as a first step, we construct a set $T \subseteq \mathrm{Z}_{6}^{4}$ which is tiling and spectral with some spectrum $L$ and further for each element $\mathbf{z} \in L^{\top}-L^{\top}$ ( $\mathbf{z}$ is 4dimensional column vector) there exists a tiling complement $R_{\mathbf{z}}$ of $T$ such that $\mathbf{z}^{\top} \notin Z\left(\widehat{\chi}_{R_{\mathbf{z}}}\right)$. Then with the help of these $R_{\mathbf{z}} \mathrm{s}$ and $L$, in the end we will construct a larger finite group $\mathscr{G}$, so that the above described situation will finally be achieved for some (other) $\mathscr{R}$ and $\mathscr{L}$.

Given a set $T$, the easiest way to produce a tiling complement of $T$ is to apply the pull-back procedure described in the next lemma.

Lemma 3 (Szegedy [14]). Let $G$ be a finite Abelian group, $T \subseteq G$ and suppose that there exists a homomorphism $\varphi: G \rightarrow H$ such that $\varphi$ is injective 
on $T$ and $\varphi(T)$ is a tile in $H$. Then $T$ tiles also $G$, and a tiling complement is given by $\varphi^{-1}(\tilde{T})$ where $\tilde{T}$ is a complement of $\varphi(T)$.

So we have to define a group homomorphism $\varphi: \mathbf{Z}_{6}^{4} \rightarrow H$ with some group $H$ such that $\varphi(T)$ tiles $H$ and $\varphi$ is injective on $T$. Then one can apply Lemma 3 to pull back the tiling complement of $\varphi(T)$ into $\mathbf{Z}_{6}^{4}$ showing $T$ to be a tile. Kolountzakis and Matolcsi [7] have applied this method with one-dimensional group homomorphisms $\varphi: Z_{6}^{5} \rightarrow Z_{6}$, in connection with a 5-dimensional decomposition of the matrix $K$. Their construction led to a counterexample in dimension 5.

To reduce the dimension to 4 we need to give a suitable 4-dimensional decomposition of $K$. The above, most straightforward, choice $K=L \cdot T$ could be a good candidate, since as remarked $T$ is spectral and also tiling. However, executing some calculations it turns out that in this case there exist some vectors $\mathbf{z} \in L^{\top}-L^{\top}$ for which there is no one-dimensional homomorphism producing a tiling complement $R^{\prime}$ of $T$ such that it satisfies the above nonvanishing requirement $\widehat{\chi}_{R^{\prime}}\left(\mathbf{z}^{\top}\right) \neq 0$.

Now, there are two possibilities, if we are sticking to Lemma 3. Either we look for non-one-dimensional homomorphisms or we choose a different $T$. Let us observe the instructive number theoretic reason of lacking such good one-dimensional $\varphi$-s: the last column of $T$ is $0 \bmod 2$. Thus we modify the above $T$ so that this obstacle vanishes. To do this, we will keep the above $L$ and $K$ and alter only $T$. Since there are only even entries in $L$, we can freely add 3 to any of the elements of $T$, while $K=L \cdot T \bmod 6$ will still hold, showing $T$ to be spectral in $\mathrm{Z}_{6}^{4}$ with the same spectrum $L$. First of all, we fix $T \bmod 3$ as in (2), so we have to specify it $\bmod 2$. Let

$$
T:=\left(\begin{array}{cccccc}
0 & 1 & 0 & 0 & 0 & 0 \\
0 & 0 & 1 & 0 & 0 & 1 \\
0 & 0 & 0 & 1 & 0 & 1 \\
0 & 0 & 0 & 0 & 1 & 1
\end{array}\right) \quad \bmod 2
$$

and hence

$$
T=\left(\begin{array}{llllll}
0 & 1 & 0 & 0 & 0 & 2 \\
0 & 0 & 1 & 0 & 0 & 5 \\
0 & 0 & 0 & 1 & 0 & 5 \\
0 & 0 & 0 & 0 & 1 & 5
\end{array}\right) \quad \bmod 6 .
$$

We claim the following

Lemma 4. Consider $T \subseteq \mathrm{Z}_{6}^{4}$ given in (3). Then for all $\mathbf{z}^{\top} \in L-L$ we find a tiling complement $R_{\mathbf{z}}$ of $T$ such that $\mathbf{z}^{\top} \notin Z\left(\widehat{\chi}_{R_{\mathbf{z}}}\right)$ and $R_{\mathbf{z}}$ is a subgroup of G. 
Proof. Let us fix $\mathbf{z}^{\top} \in L-L$. Our idea, as described in the preceding discussion, is to produce the tiling complement $R_{\mathbf{z}}$ as $\operatorname{ker} \varphi$ for some onedimensional homomorphism, i.e., we look for the homomorphism in the form $\varphi(\mathbf{x})=\mathbf{v}^{\top} \cdot \mathbf{x}$ with some $\mathbf{v} \in \mathrm{Z}_{6}^{4}$ to be chosen appropriately (column vector, hence $\mathbf{v}^{\top}$ is a row vector). Then for the Fourier transform

$$
\widehat{\chi}_{R_{\mathbf{z}}}\left(\mathbf{w}^{\top}\right)=\sum_{\mathbf{x} \in R_{\mathbf{z}}} e^{\frac{2 \pi i}{6} \mathbf{w}^{\top} \cdot \mathbf{x}}
$$

and a choice of $\mathbf{v}$ satisfying $\alpha \mathbf{v}=\mathbf{z}$ with some $\alpha \in \mathbf{Z}_{6}$ will ensure

$$
\widehat{\chi}_{R_{\mathbf{z}}}\left(\mathbf{z}^{\top}\right)=\sum_{\mathbf{x} \in R_{\mathbf{z}}} e^{\frac{2 \pi i}{6} \alpha \mathbf{v}^{\top} \cdot \mathbf{x}}=\sum_{\mathbf{x} \in R_{\mathbf{z}}} e^{\frac{2 \pi i}{6} \alpha \varphi(\mathbf{x})}=\sum_{\mathbf{x} \in R_{\mathbf{z}}} e^{0}=\left|R_{\mathbf{z}}\right|>0 .
$$

So the homomorphism $\varphi$ should be given in such a way that $\mathbf{z}^{\top}$ becomes a scalar multiple of $\mathbf{v}^{\top}$.

To find a suitable $\mathbf{v}$ we let $\mathbf{k}:=\mathbf{z}^{\top} \cdot T \in K-K$. Notice that $\mathbf{z}$ has even coordinates, and $\mathbf{k}$ is a permutation of $(0,0,2,2,4,4) \bmod 6$. Now "divide" $\mathbf{k}$ by $2(\bmod 6)($ this is because of the above consideration with $\alpha)$; then for each entry we have two possibilities, as $0=2 \cdot 0=2 \cdot 3,2=2 \cdot 1=2 \cdot 4$, $4=2 \cdot 2=2 \cdot 5$.

So we fix e among the possible "halves" of $\mathbf{k}$ such that it will be a permutation of $(0,1,2,3,4,5)$ and, moreover that the matrix equation $\mathbf{v}^{\top} \cdot T=\mathbf{e}$ has a solution $\bmod 6$ in $\mathbf{v}$. Actually, it is enough to solve $\mathbf{v}^{\top} \cdot T=\mathbf{e} \bmod 3$ and $\bmod 2$, and then the mod 6 solution is easily recovered. These assumptions will ensure that the homomorphism $\varphi: \mathbf{Z}_{6}^{4} \rightarrow \mathbf{Z}_{6}$ defined by $\mathbf{v}$ is surjective (hence tiling) and injective on $T$. Observe that $K-K$ consists of all the vectors with first coordinate 0 and the rest 5 coordinates being any permutation of $(0,2,2,4,4)$. Thus for any choice of $\mathbf{e}$, by $2 \cdot 2=1 \bmod 3$ we will have $\mathbf{e}=2 \mathbf{k} \bmod 3$. That is, a solution $\mathbf{v}_{3}$ of $\mathbf{v}^{\top} \cdot T=\mathbf{e} \bmod 3$ undoubtedly exists, because $L \cdot T=K \bmod 3$, hence $2 \cdot(L-L) \cdot T$ covers $2(K-K)$ containing $\mathbf{e}=2 \mathbf{k} \bmod 3$. We show that with an appropriate choice of $\mathbf{e}$ one also finds a mod 2 solution. Clearly the first coordinate $e_{1}$ of $\mathbf{e}=\left(e_{1}, e_{2}, e_{3}, e_{4}, e_{5}, e_{6}\right)$ can be fixed as 0 . Among the coordinates of $\mathbf{k}$ there are exactly two falling into each of the $\bmod 3$ classes. These we call pairs. Now we have to distinguish between these pairs mod 2 . Notice that we can choose e such that among $e_{2}, e_{3}$ and $e_{4}$ exactly two are odd. Indeed, among these three elements there is either a pair from the same mod 3 class, or all three elements differ mod 3 . In either case we can prescribe $e_{2}, e_{3}$ and $e_{4}$ such that $e_{2}=1 \bmod 2$, and $e_{3}$ and $e_{4}$ have different parity, while for the rest two coordinates $e_{5}$ and $e_{6}$ of $\mathbf{e}$ the only restriction is that the mod 3 pairs have to be mod 2 different. Choosing e in such a way and using (2) an easy 
calculation shows that $\mathbf{v}^{\top} \cdot T=\left(0, v_{1}, v_{2}, v_{3}, v_{4}, v_{2}+v_{3}+v_{4}\right)=\mathbf{e} \bmod 2$ has a solution $\mathbf{v}_{2} \bmod 2$.

Now the desired $\mathbf{v}$ can be computed from $\mathbf{v}_{2}$ and $\mathbf{v}_{3}$ because the moduli are relatively primes.

It remains only to show that $\mathbf{z}^{\top} \notin Z\left(\widehat{\chi}_{R_{z}}\right)$, but this is obvious by construction. In fact, let $\mathbf{x} \in R_{\mathbf{z}}$ : this means $\mathbf{v}^{\top} \cdot \mathbf{x}=0$. On the other hand, $2 \mathbf{v}^{\top} \cdot T=2 \mathbf{e}$ and $\mathbf{z}^{\top} \cdot T=\mathbf{k}=2 \mathbf{e}$. From this $2 \mathbf{v}=\mathbf{z}$ follows, whence $0=2 \mathbf{v}^{\top} \cdot \mathbf{x}=\mathbf{z}^{\top} \cdot \mathbf{x}$, so keeping (5) in mind gives $\widehat{\chi}_{R_{z}}\left(\mathbf{z}^{\top}\right)>0$.

REMARK 3. Let us make the above proof more comprehensible by means of a particular example of constructing $\mathbf{v}$ and the corresponding homomorphism. E.g., let $\mathbf{z}^{\top}:=(0,2,2,4) \in L-L$. Then $\mathbf{k}=\mathbf{z}^{\top} \cdot T=(0,0,2,2,4,4) \bmod 6$. So $\mathbf{e}=\mathbf{k} / 2=(0,0,1,1,2,2) \bmod 3$, and as described above we can choose $\mathbf{e}=(0,1,1,0,1,0) \bmod 2$, resulting in $\mathbf{e}=(0,3,1,4,5,2) \bmod 6$. The solution vectors $\mathbf{v}_{2}$ and $\mathbf{v}_{3}$ are $\mathbf{v}_{2}^{\top}=(1,1,0,1) \bmod 2$ and $\mathbf{v}_{3}^{\top}=(0,1,1,2) \bmod 3$, hence $\mathbf{v}^{\top}=(3,1,4,5)$.

Using the above $T$, its tiling complements $R_{\mathbf{z}}:=\operatorname{ker} \varphi$ (with the $\varphi$ above depending on $\mathbf{z}$ ) and also its spectrum $L$, we are now in the position to construct our final counterexample to the "tiling $\Rightarrow$ spectral" part of Fuglede's Conjecture in dimension 4.

Proof of Theorem 1. Let $L^{\top}-L^{\top}=\left\{\mathbf{z}_{j}: j=1, \ldots, k\right\}$, say $\left(\mathbf{z}_{j}\right.$ is a column vector). Take $\mathscr{L} \subseteq \widehat{\mathscr{G}}:=\mathrm{Z}_{6}^{4} \times \mathrm{Z}_{p}$ to be the set of the elements of $L$ extended by a 0 in the fifth coordinate (i.e., considering $L \subseteq \widehat{G} \cong$ $\widehat{G} \times\{0\}=: \widehat{\mathscr{G}}_{0}$ as imbedded into $\mathscr{G}$, which trivial identification - as well as the similar, dual imbedding of $G$ into $\mathscr{G}$ - we do not mention further on). We put together the desired tiling but not spectral set from the above constructed tiling complements $R_{j}:=R_{\mathbf{z}_{j}}$ of $T \times\{0\}$. So let $p \geq k$ be relatively prime to 6 , and let us augment the sequence $R_{1}, \ldots, R_{k}$ by listing the $R_{j}$ s and then repeating $R_{k}$ additionally $p-k$ times. Consider the group $\mathscr{G}=\mathrm{Z}_{6}^{4} \times \mathrm{Z}_{p}$ (which is, on the other hand, isomorphic to $Z_{6}^{3} \times Z_{6 p}$ ) and the set

$$
\mathscr{R}=\bigcup_{j=1}^{p}\left(R_{j}+(0,0,0,0, j)^{\top}\right) .
$$

Consider now the sets $\mathscr{R}$ and $\mathscr{L}$. First, $\mathscr{R}+T \times\{0\}$ is a tiling, as for all $j=1, \ldots, p\left(R_{j}+(0,0,0,0, j)^{\top}\right)+T \times\{0\}$ is a tiling of the translated subgroups $\mathscr{G}_{0}+(0,0,0,0, j)^{\top}$ of $\mathscr{G}_{0}:=\mathrm{Z}_{6}^{4} \times\{0\}$. Hence $\mathscr{R}$ is a tile of $\mathscr{G}$ with the tiling complement $T \times\{0\}$.

Moreover, $L$ is a spectrum of $T$, hence we get $|\mathscr{L}|=|L|=|T|=|\mathscr{G}| /|\mathscr{R}|$. (It can also bee seen easily that $\mathscr{L}$ is a spectrum of $T \times\{0\}$, but we do not 
need this here.) We need to show that also $Z\left(\widehat{\chi}_{\mathscr{R}}\right) \cap(\mathscr{L}-\mathscr{L})=\emptyset$. So let $0 \neq \mathbf{z} \in \mathscr{L}^{\top}-\mathscr{L}^{\top}$ be any element; it corresponds to $\mathbf{z}_{j}$ for some index $j \leq p$. Then the Fourier transform of $\chi_{\mathscr{R}}$ evaluated at $\mathbf{z}^{\top}$ is

$$
\widehat{\chi}_{\mathscr{R}}\left(\mathbf{z}^{\top}\right)=\widehat{\chi}_{R_{1}}\left(\mathbf{z}^{\top}\right)+\cdots+\widehat{\chi}_{R_{k-1}}\left(\mathbf{z}^{\top}\right)+(p-k+1) \widehat{\chi}_{R_{k}}\left(\mathbf{z}^{\top}\right)>0,
$$

because all the terms are non-negative (all $R_{m}$ s being subgroups), and by construction the $j^{\text {th }}$ term is strictly positive in view of Lemma 4 . So $\mathscr{R}$ and $\mathscr{L}$ fulfill the initial requirements for a pair of sets for a counterexample.

Furthermore, $\mathscr{L}$ is not a tile. To see this note that $\mathscr{L} \subset \widehat{\mathscr{G}}_{0}$, hence $\mathscr{L}$ can be a tile if only it tiles also the subgroup $\widehat{\mathscr{G}}_{0}$, that is, if $L$ tiles $\widehat{G}$. But since $L$ consists of vectors with all coordinates even, it is in fact a subset of the subgroup $E \leq \widehat{G}$ with even coordinates, hence in order to tile $\widehat{G}$, it has to tile even $E$. However, this is not possible since $|L|=6$, which does not divide $|E|=3^{4}$. Thus we see that the sets $\mathscr{R}$ and $\mathscr{L}$ provide all the properties of the construction we were aiming at, whence $\mathscr{R}$ is tiling $\mathscr{G}$ while being non spectral.

Having a counterexample in $\mathscr{G} \cong \mathrm{Z}_{6}^{3} \times \mathrm{Z}_{6 p}$, the counterexample in $\mathrm{Z}^{4}$ and $\mathrm{R}^{4}$ is obtained by an application of Theorems 2 and 3 .

\section{REFERENCES}

1. Fuglede, B., Commuting self-adjoint partial differential operators and a group theoretic problem, J. Funct. Anal. 16 (1974), 101-121.

2. Iosevich, A., Katz, N., Tao, T., Convex bodies with a point of curvature do not admit exponential bases, Amer. J. Math. 123 (2001), 115-120.

3. Iosevich, A., Katz, N., Tao, T., The Fuglede spectral conjecture holds for convex bodies in the plane, Math. Res. Lett. 10 (2003), 559-570.

4. Kolountzakis, M. N., Non-symmetric convex domains have no basis of exponentials, Illinois J. Math. 44 (2000), no. 3, 542-550.

5. Kolountzakis, M. N., The study of translational tiling with Fourier Analysis in Proceedings of the Workshop on Fourier Analysis and Convexity, 131-187, Appl. Numer. Harmon. Anal., 2004.

6. Kolountzakis, M. N., Matolcsi, M., Complex Hadamard matrices and the Spectral Set Conjecture, Collect. Math., to appear.

7. Kolountzakis, M. N., Matolcsi, M., Tiles with no spectra, Forum Math, to appear.

8. Konyagin, S., I. Łaba, Spectra of certain types of polynomials and tiling of integers with translates of finite sets, J. Number Theory 103 (2003), no. 2, 267-280.

9. Lagarias, J. C., and Wang, Y., Spectral sets and factorizations of finite Abelian groups, J. Funct. Anal. 145 (1997), 73-98.

10. Lagarias, J. C., Szabó, S., Universal spectra and Tijdeman's conjecture on factorization of cyclic groups, J. Fourier Anal. Appl. 7 (2001), no. 1, 63-70.

11. Matolcsi, M., Fuglede's conjecture fails in dimension 4, Proc. Amer. Math. Soc. 133 (2005), 3021-3026.

12. Pedersen, S., Wang, Y., Universal spectra, universal tiling sets and the spectral set conjecture, Math. Scand. 88 (2001), no. 2, 246-256. 
13. Rudin, W., Fourier Analysis on Groups, Reprint of the 1962 original, Wiley Classics Lib., 1990.

14. Szegedy, M., Algorithms to Tile the Infinite Grid with Finite Clusters, FOCS 1998, pp. 137147.

15. Tao, T., Fuglede's conjecture is false in 5 and higher dimensions, Math. Res. Lett., 11 (2004), no. 2-3, 251-258.

TECHNISCHE UNIVERSITÄT DARMSTADT

FACHBEREICH MATHEMATIK AG 4

SCHLOßGARTENSTRAßE 7

D-64289 DARMSTADT

GERMANY

E-mail: farkas@mathematik.tu-darmstadt.de

\author{
ALFRÉD RÉNYI INSTITUTE \\ HUNGARIAN ACADEMY OF SCIENCES \\ REÁLTANODA U. 13-15 \\ H-1053, BUDAPEST \\ HUNGARY \\ E-mail: revesz@renyi.hu
}

